\title{
Glycemic Benefits with Adherence to testosterone therapy in men with hypogonadism and type 2 diabetes mellitus.
}

Craig R. Jenkins

Brooke Army Medical Center

Alex Rittel

Defense Healthcare Management Systems

Rodney X. Sturdivant

Henry M. Jackson Foundation for the Advancement of Military Medicine

Jen Wan

Brooke Army Medical Center

Philip G. Clerc

Brooke Army Medical Center

See next page for additional authors

Tell us how you used this information in this short survey.

Follow this and additional works at: https://digitalcommons.unmc.edu/com_hosp_articles

\section{Recommended Citation}

Jenkins, Craig R.; Rittel, Alex; Sturdivant, Rodney X.; Wan, Jen; Clerc, Philip G.; Manning, Evan; Jenkins, Lydia M.; Wardian, Jana L. PhD; and Graybill, Sky D., "Glycemic Benefits with Adherence to testosterone therapy in men with hypogonadism and type 2 diabetes mellitus." (2021). Journal Articles: Hospital Medicine. 2.

https://digitalcommons.unmc.edu/com_hosp_articles/2 


\section{Authors}

Craig R. Jenkins, Alex Rittel, Rodney X. Sturdivant, Jen Wan, Philip G. Clerc, Evan Manning, Lydia M. Jenkins, Jana L. Wardian PhD, and Sky D. Graybill 


\title{
Glycemic benefits with adherence to testosterone therapy in men with hypogonadism and type 2 diabetes mellitus
}

\author{
Craig R. Jenkins ${ }^{1}$ (0) | Alex Rittel ${ }^{2}$ | Rodney X. Sturdivant ${ }^{3}$ ～Jen Wan ${ }^{1}$ | Philip G. Clerc ${ }^{1}$ | \\ Evan Manning $^{4}$ | Lydia M. Jenkins ${ }^{5}$ | Jana L. Wardian ${ }^{6}$ | Sky D. Graybill ${ }^{1}$ @
}

${ }^{1}$ Department of Medicine, Endocrinology Service, Brooke Army Medical Center, Ft Sam Houston, TX, USA

${ }^{2}$ Data Innovation Branch, Defense Healthcare Management Systems, San Antonio, TX, USA

${ }^{3}$ Henry M. Jackson Foundation for the Advancement of Military Medicine, Bethesda, MD, USA

${ }^{4}$ Department of Medicine, Internal Medicine, Brooke Army Medical Center, Ft Sam Houston, TX, USA

${ }^{5}$ Department of Pediatrics, Brooke Army Medical Center, Ft Sam Houston, TX, USA

${ }^{6}$ Diabetes Center of Excellence, Wilford Hall Ambulatory Surgical Center, Lackland AFB, TX, USA

\section{Correspondence}

Craig R. Jenkins, Department of Medicine, Endocrinology Service, Brooke Army Medical Center, Ft Sam Houston, Texas, USA.

Email: craig.r.jenkins2.mil@mail.mil

\begin{abstract}
Background: While previous studies have demonstrated testosterone's beneficial effects on glycemic control in men with hypogonadism and Type 2 Diabetes, the extent to which these improvements are observed based on the degree of treatment adherence has been unclear.
\end{abstract}

Objectives: To evaluate the effects of long-term testosterone therapy in $\mathrm{A} 1 \mathrm{C}$ levels in men with Type 2 Diabetes Mellitus and hypogonadism, controlling for BMI, pretreatment $\mathrm{A} 1 \mathrm{C}$, and age among different testosterone therapy adherence groups.

Materials and methods: We performed a retrospective analysis of 1737 men with diabetes and hypogonadism on testosterone therapy for 5 years of data from 2008 2018, isolating A1C, lipid panels, and BMI results for analysis. Subjects were categorized into adherence groups based on quartiles of the proportion of days covered (> $75 \%$ of days, $51-75 \%$ of days, $26-50 \%$ of days and $0-25 \%$ of days), with $>75 \%$ of days covered considered adherent to therapy.

Results: Pre-treatment median A1C was 6.8\%. Post-treatment median A1C was $7.1 \%$. The adherent group, $>75 \%$, was the only group notable for a decrease in $\mathrm{A} 1 \mathrm{C}$, with a median decrease of -0.2 ( $p=0.0022$ ). BMl improvement was associated with improved post-treatment A1C ( $p=0.007)$. When controlling for BMI, age, and pre-treatment A1C, the $>75 \%$ adherence group was associated with improved post-treatment A1C $(p<0.001)$.

Discussion: When controlling for all studied variables, testosterone adherence was associated with improved post-treatment $\mathrm{A} 1 \mathrm{C}$. The higher the initial $\mathrm{A} 1 \mathrm{C}$ at the initiation of therapy, the higher the potential for lowering the patient's $\mathrm{A} 1 \mathrm{C}$ with $>75 \%$ adherence. Further, all groups showed some reduction in BMI, which may indicate that testosterone therapy may affect $\mathrm{A} 1 \mathrm{C}$ independent of weight loss.

Conclusion: Even when controlling for improved BMI, pre-treatment A1C, and age, testosterone positively impacted glycemic control in diabetes patients with hypogonadism, with the most benefit noted in those most adherent to therapy ( $>75 \%)$.

KEYWORDS

hypogonadism, testosterone treatment, type 2 diabetes 


\section{INTRODUCTION}

In the United States, type 2 diabetes mellitus (T2DM) and prediabetes prevalence has grown in recent decades, with a total burden of nearly $40.0 \%$ for patients 20 years of age or older. ${ }^{1}$ Lifetime risk of progression to diabetes from pre-diabetes is reported as high as $74.0 \%{ }^{2}$ Hypogonadism is likewise common, with prevalence estimated at $38.7 \%$ for males over the age of $45 .^{1}$

Approximately $25-33 \%$ of men with T2DM have hypogonadotropic hypogonadism, ${ }^{3}$ and hypogonadism has been suggested as an independent risk factor for the development of T2DM. ${ }^{4}$ There is a growing and sometimes unclear body of research investigating whether testosterone should be utilized as part of the standard clinical management of T2DM. Some small studies have demonstrated favorable effects of testosterone replacement therapy on insulin sensitivity and hemoglobin $\mathrm{A} 1 \mathrm{C}(\mathrm{A} 1 \mathrm{C})$ in men with T2DM,${ }^{5-7}$ whereas others showed no benefit. ${ }^{8,9}$

One meta-analysis of randomized controlled trial on men with T2DM and/or metabolic syndrome found no evidence of an improvement in $\mathrm{HbA} 1 \mathrm{c}$ with testosterone replacement therapy, ${ }^{10}$ while the benefits of testosterone therapy in improving glycemic control and insulin resistance has been observed in some other studies. One study found that in men with Type 2 DM and hypogonadism, those on testosterone therapy (vs. on standard diabetes management alone) enjoyed improvements in fasting glucose, HbA1c, and fasting insulin over the 11 year treatment period, and among the patients treated with testosterone, $34.3 \%$ achieved remission of their diabetes and $46.6 \%$ of patients achieved normal glucose regulation. Of the testosterone-treated group, $83.1 \%$ reached the $\mathrm{HbA} 1 \mathrm{c}$ target of $47.5 \mathrm{mmol} / \mathrm{mol}$ (6.5\%) and $90 \%$ achieved the $\mathrm{HbA1c}$ target of $53.0 \mathrm{mmol} / \mathrm{mol}$ (7\%). In the control group, no remission of diabetes or reductions in $\mathrm{HbA} 1 \mathrm{c}$ levels were noted. ${ }^{11}$ Other studies have replicated these findings, showing long-term testosterone therapy, defined as being on therapy for 8 years, completely prevented progression to T2DM in men with hypogonadism. ${ }^{12}$

The relationship between androgens, glycemia, and weight loss is complex and interrelated. Prior studies have investigated the relationship between androgen levels and glycemic control in patients with hypogonadism and type $2 \mathrm{DM}$. One study demonstrated that in obese men with hypogonadism and uncontrolled type 2 diabetes mellitus, weight loss seemed to have a greater impact on final testosterone levels than improved glycemic control. ${ }^{13}$ When adjusting for $\mathrm{BMI}$, age, waist circumference and metabolic syndrome, men with pre-diabetes are almost twice as likely to have low testosterone levels than their counterparts with euglycemia. ${ }^{14}$ In 2018, the American Diabetes Association (ADA) $)^{15}$ added a recommendation to measure testosterone in men with T2DM and signs/symptoms of hypogonadism. Acknowledging that the relationship between androgens, glycemia, and weight loss is complex and interrelated, our study attempted to provide more data on the impact of testosterone therapy on glycemic control and weight loss in the same subset of patients.

The United States military is not an exception to the above trends, with incidence of T2DM comparable to that of the US population as a whole ${ }^{16}$ although data are currently lacking for hypogonadism. There is a need for further investigation and treatment of both conditions for the population as a whole and for the military in particular, as both hypogonadism and T2DM are associated with an increased risk of cardiovascular disease (CVD) and all-cause mortality ${ }^{17}$ as well as other comorbidities. ${ }^{18}$ In addition, T2DM has been associated with reduced employment and increased work limitations, which may have important implications for military readiness. ${ }^{19}$

Effective interventions may provide improvements in patient health and in workplace participation. Currently, The American Association of Clinical Endocrinologist Clinical Practice guidelines recommend that patients with obesity, overweight, and/or prediabetes achieve a weight loss of $10 \%$ to prevent progression to T2DM. ${ }^{20,21}$ Even 10 and 15 years later, lifestyle interventions reduced T2DM onset by $34 \%$ and $27 \%$, respectively, and delayed the onset of T2DM by about 4 years compared with placebo. ${ }^{20,21}$ Our study aims to evaluate the effects of long-term testosterone therapy on $\mathrm{A} 1 \mathrm{C}$ in men with co-occurring T2DM and hypogonadism in our DoD beneficiary population, with the hypothesis that the men most adherent to therapy will have improved glycemia over time.

\section{2 | MATERIALS AND METHODS}

\subsection{Data Sources}

Data was collected from the Military Health System (MHS) Management Analysis and Reporting Tool, which contains health care service data provided by military treatment facilities (MTFs), civilian providers, hospitals, managed care support contractors, the Defense Manpower Data Center, and the Pharmacy Data Transaction Service, including inpatient, outpatient, emergency department (ED), ancillary, pharmaceutical, financial, and enrollment data. Laboratory data (A1C, lipid panels) came from the MHS Composite Health Care System (CHCS). This study protocol was approved by the Department of Defense Review Board and TRICARE Management Activity Human Protection and Privacy Department before data collection.

\subsection{Study design}

This was a retrospective study of 1737 men with comorbid diabetes and hypogonadism on testosterone replacement therapy from 2008-2018. The electronic medical record was utilized to identify individuals with comorbid diabetes and hypogonadism diagnoses occurring prior to 2014 to allow 5 years of follow-up data. The date of initial hypogonadism diagnosis was used as the start date and the date 5 years after the initial hypogonadism diagnosis was used as the follow-up date. Laboratory and vitals were queried to isolate one data point for $\mathrm{A} 1 \mathrm{C}$, lipid panel, and $\mathrm{BMI}$ results occurring in the 12 months prior to the start date and in the 12 months following the follow-up date. If there was more than 1 data point available, we 
utilized the value most proximal in time frame to our study. For example, if an A1C value fell out of the established threshold (between $4 \%$ and $12 \%$ ), this triggered a chart review by a physician to ensure that this $\mathrm{A} 1 \mathrm{C}$ value was not an outlier for that specific patient given their history, and hence a likely lab error. If this was the case, the A1C value was omitted, and the next proximal value to the study was utilized. A similar approach was also used with lipids and BMI. We omitted lipid panels when LDL was "unable to be calculated," which was usually due to hypertriglyceridemia $>400 \mathrm{mg} / \mathrm{dL}$, and if BMI values were obvious outliers for that patient, and thus, likely a recording error. Pharmacy data records were used to determine all prescriptions for testosterone replacement therapy in the 5 years following the start date and a proportion of days covered calendar was created for all patients to define adherence to medication. Patients were not excluded from the study based on the formulation of testosterone they were prescribed by their provider. However, the majority of patients were given with transdermal testosterone or testosterone cypionate. Eligibility criteria included adult males (ages 18-75) with a diagnosis of type 2 diabetes mellitus at the time of initial hypogonadism diagnosis, at least one day of testosterone replacement therapy coverage in the 5 years following initial hypogonadism diagnosis, and pre-/post-A1C results. Exclusion criteria included a history of metabolic surgery, evidence of testosterone replacement therapy prior to the initial hypogonadism diagnosis, and initial hypogonadism diagnoses occurring after Jan 1, 2014.

\section{3 | Statistical analysis}

1737 adult men were identified with comorbid diabetes and hypogonadism with testosterone replacement therapy during the specified time interval. Subjects were categorized by age group and into their respective adherence groups based on their prescription refill activity. For example, if the subject refilled his testosterone $>75 \%$ of the time it was prescribed for, they were placed in the $>75 \%$ adherence group. If they refilled their prescription so that $51-75 \%$ of expected days were covered, they were placed in the $51-75 \%$ group. The same followed for the $26-50 \%$ group, and the $0-25 \%$ groups. $>75 \%$ proportion of days covered was considered adherent to therapy. The primary goal of this study was to assess the difference in pre-/postA1C among the various adherence groups and to determine if individuals in the highest adherence group had improvements in other clinical outcome measures, including BMI and cholesterol (total, LDL and $\mathrm{HDL}$ ).

Multivariate linear models were used to determine the effect of treatment adherence on post-treatment $\mathrm{A} 1 \mathrm{C}$ controlling for potential confounding variables. Model variables were selected by the method of purposeful selection. ${ }^{22}$ The recommended "ANCOVAPOST" approach of O'Connell et al. ${ }^{23}$ for analyzing pre-post was used with post-treatment A1C (transformed to 1/A1C_Post) as the response and pre-treatment $A 1 C$ as a predictor in the model. Due to issues with the normality and equal variance assumptions of the model, a Box-Cox transformation analysis ${ }^{24}$ was performed producing an optimal power of $\lambda=-1 \cdot 3 \cdot \lambda=-1$ was chosen to transform the response as it produced more easily interpretable results while adequately addressing issues with model assumptions. Multiple Imputation by Chained Equations ${ }^{25}$ was utilized to address missing BMI and cholesterol readings. Five imputed data sets were utilized as recommended by Rubin ${ }^{26}$ and the results of fitting models to these data sets combined using the rules outlined in the same reference. The lowest adherence group ( $0-25 \%$ of days covered) and the youngest age group ( $<45$ years old) were used as reference groups with reference cell coding for the categorical predictors.

Descriptive analysis compared the values of A1C (posttreatment, pre-treatment and the change (post minus pre)) for categorical variables using medians, the interquartile range (IQR), and Kruskal-Wallis test for the change. This was due to lack of normality within groups. For continuous variables such as BMI and cholesterol measurements, means, standard deviations and results of separate regression models with transformed post-treatment $\mathrm{A} 1 \mathrm{C}$ (1/A1C_Post) as the response and pre-treatment A1C controlled for in the model were fit using only subjects with observed values for a particular measure (ie, complete case analysis). $P$-values $<0.05$ were considered significant.

All analyses were performed in RStudio version 1.2.1335.

\section{3 | RESULTS}

The median A1C for all 1737 men in the study was $6.8 \%(I Q R=1.5)$ pre-treatment and 7.1\% (IQR $=1.8)$ post-treatment. Table $1 \mathrm{dem}$ onstrates that the increase in $\mathrm{A} 1 \mathrm{C}$ was greatest in men in the lowest adherence group, with a median increase of 0.2 and 0.19 in the $0-25 \%$ and $26-50 \%$ adherence groups, respectively. The adherent group, $>75 \%$, was the only group notable for a decrease in $\mathrm{A} 1 \mathrm{C}$, with a median change of -0.2 . Table 1 also demonstrates that posttreatment $\mathrm{A} 1 \mathrm{C}$ does differ with adherence groups $(p=0.0022)$ and age group ( $p=0.0008$ ). For example, the results suggest that the differences in $\mathrm{A} 1 \mathrm{C}$ change in the adherence groups are in the $75 \%+$ group, but since this was a univariate analysis, this did not control for other factors, which we addressed with a multivariate analysis below.

Next, we performed a separate univariate regression analysis to see if other clinical measures (BMI and cholesterol measurements including total, $\mathrm{LDL}$, and $\mathrm{HDL}$ ) were related to $\mathrm{A} 1 \mathrm{C}$ change, but controlling for pre-treatment $\mathrm{A} 1 \mathrm{C}$ alone. The results are listed in Table 2 Notably, cholesterol measurements, in particular, are missing for a large percentage of men in the study, but this was addressed with multiple imputation by chained equations, as addressed in the statistical analysis section. We found that, for example, pre-treatment $\mathrm{BMI}$ does not seem to be related to a change in $\mathrm{A} 1 \mathrm{C}$ from pre- to post-treatment $(p=0.801)$. Factors that did seem related to a change in A1C, however, were HDL levels post-treatment ( $p=0.031$ ), HDL change from post-treatment to pre-treatment ( $p=0.018)$, cholesterol post-treatment $(p<0.001)$, and cholesterol change from posttreatment to pre-treatment $(p<0.001)$. This was a univariate analysis 
TAB LE 1 Descriptive Statistics for A1C, Overall and by Adherence and Age Group ( $P$ Value for A1C Change)

\begin{tabular}{|c|c|c|c|c|c|}
\hline & $\mathrm{n}$ & $\begin{array}{l}\text { Pre-treatment A1C median } \\
\text { (IQR) }\end{array}$ & $\begin{array}{l}\text { Post-treatment A1C } \\
\text { median (IQR) }\end{array}$ & $\begin{array}{l}\text { A1C Change (Post-Pre) } \\
\text { median (IQR) }\end{array}$ & $P$ Value \\
\hline Overall & 1737 & $6.80(1.50)$ & $7.10(1.80)$ & $0.20(1.50)$ & \\
\hline \multicolumn{6}{|c|}{ Adherence Group (\% days) } \\
\hline $0-25$ & 1097 & $6.80(1.60)$ & $7.20(2.00)$ & $0.20(1.60)$ & \multirow[t]{4}{*}{.0022} \\
\hline $26-50$ & 337 & $6.80(1.30)$ & $7.10(1.70)$ & $0.19(1.50)$ & \\
\hline $51-75$ & 212 & $6.65(1.40)$ & $6.80(1.40)$ & $0.10(1.22)$ & \\
\hline$>75$ & 91 & $6.70(1.25)$ & $6.60(1.15)$ & $-0.20(1.01)$ & \\
\hline \multicolumn{6}{|c|}{ Age Group (years) } \\
\hline$<45$ & 103 & $6.90(2.15)$ & $7.70(2.70)$ & $0.40(2.10)$ & \multirow[t]{3}{*}{.0008} \\
\hline $45-59$ & 1042 & $6.80(1.70)$ & $7.30(1.90)$ & $0.20(1.68)$ & \\
\hline $60-75$ & 592 & $6.70(1.30)$ & $6.80(1.40)$ & $0.05(1.20)$ & \\
\hline
\end{tabular}

Note: The column showing $\mathrm{A} 1 \mathrm{C}$ change involves first taking the differences (post-pre) for all subjects and then computing the median of those differences. The reason to show this number is that we model the individual differences not the overall difference in medians.

\begin{tabular}{|lccccc|} 
& $\begin{array}{l}\text { Missing } \\
\text { Values }\end{array}$ & Mean (SD) & Coefficient & $\begin{array}{l}\text { Std. } \\
\text { Error }\end{array}$ & P Value \\
\hline BMI Pre-treatment & 36 & $33.9(5.7)$ & -0.002 & 0.006 & .801 \\
\hline BMI Post-treatment & 89 & $33.5(6.0)$ & 0.003 & 0.006 & .639 \\
\hline BMI Change (Post - Pre) & 110 & $-0.4(3.7)$ & 0.019 & 0.01 & .052 \\
\hline HDL Pre-treatment & 906 & $39.1(10.9)$ & -0.0001 & 0.005 & .979 \\
\hline HDL Post-treatment & 1245 & $39.9(12.1)$ & -0.014 & 0.006 & .031 \\
\hline HDL Change (Post - Pre) & 1404 & $0.5(8.9)$ & -0.026 & 0.011 & .018 \\
\hline LDL Pre-treatment & 918 & $114.2(31.3)$ & 0.002 & 0.002 & .185 \\
\hline LDL Post-treatment & 1251 & $116.0(34.2)$ & 0.001 & 0.002 & .700 \\
\hline LDL Change (Post - Pre) & 1417 & $-0.1(35.6)$ & 0.001 & 0.003 & .679 \\
\hline CHOL Pre-treatment & 898 & $196.0(39.8)$ & 0.002 & 0.001 & .095 \\
\hline CHOL Post-treatment & 1245 & $197.5(41.3)$ & 0.007 & 0.002 & $<.001$ \\
\hline CHOL Change (Post - Pre) & 1402 & $-2.5(49.1)$ & 0.007 & 0.002 & $<.001$ \\
\hline
\end{tabular}

TABLE 2 Summary statistics and results of separate regressions of BMI and cholesterol measurements on transformed post-treatment A1C (1/A1C_Post) (controlling for pre-treatment $\mathrm{A} 1 \mathrm{C}$ in each model, complete case analysis)

\begin{tabular}{|lllll|} 
& Coefficient & Std. Error & $95 \%$ Cl & $P$ Value \\
\hline Intercept & 0.178 & 0.003 & $(0.17,0.19)$ & $<.001$ \\
\hline A1C_Pre-treatment & -0.007 & 0.0003 & $(-0.0076,-0.0062)$ & $<.001$ \\
\hline Adherence Group 26-50 & 0.002 & 0.0014 & $(-0.0003,0.0051)$ & .093 \\
\hline Adherence Group 51-75 & 0.005 & 0.0017 & $(0.002,0.009)$ & .002 \\
\hline Adherence Group $>75$ & 0.011 & 0.0025 & $(0.006,0.016)$ & $<.001$ \\
\hline Age Group 45-59 & 0.007 & 0.002 & $(0.003,0.012)$ & .002 \\
\hline Age Group 60-75 & 0.014 & 0.002 & $(0.009,0.018)$ & $<.001$ \\
\hline BMI Change (Post - Pre) & -0.0004 & 0.0002 & $(-0.0007,-0.0001)$ & .007 \\
\hline
\end{tabular}

TABLE 3 Multivariate Model of transformed Post-treatment A1C (1/A1C_ Post), using MICE for missing BMI data

Note: These are the combined estimates, using Rubin's rules, based on models fit to multiply imputed data sets using MICE. The average $\mathrm{R}^{2}$ for the models fit to imputed data sets is 0.24 (95\% $\mathrm{Cl}: 0.20-0.27)$ reflecting large individual variability in results.

controlling only for pre-treatment $\mathrm{A} 1 \mathrm{C}$, and not controlling for other variables, so we moved now to a multivariate analysis.

Table 3 represents the final multivariable regression model, ultimately controlling for all variables. This model demonstrates that when controlling for pre-treatment $\mathrm{A} 1 \mathrm{C}$, adherence group, and age group, BMI change was associated with improved post-treatment A1C ( $p=0.007)$. And further, notably for our study, even when controlling for BMI, age group, and pre-treatment $\mathrm{A} 1 \mathrm{C}$, the $>75 \%$ 
testosterone adherence group was associated with improved posttreatment $\mathrm{A} 1 \mathrm{C}(p<0.001)$.

The estimates shown are the combined estimates, using Rubin's rules, based on models fit to multiply imputed data sets using MICE. The average $\mathrm{R}^{2}$ for the models fit to imputed data sets is 0.24 (95\% $\mathrm{Cl}$ : 0.20-0.27) reflecting large individual variability in results. Issues with model assumptions (constant variance, normality) were successfully addressed using the transformed response variable based on residual analysis (not shown). The pre-treatment $\mathrm{A} 1 \mathrm{C}$ was controlled for as a predictor in the model in univariate analysis. Adherence group, age group and BMI change were all significant predictors included in the final multivariate model. The least adherent group $(0-25 \%)$ and youngest group ( $<45$ years old) were reference groups. Interaction effects were tested but none were significant.

Interpreting the model, the plot in Figure 1 is a visual representation of the model in Table 3 which controls for BMI, age, and pre-treatment $\mathrm{A} 1 \mathrm{C}$ value, demonstrating the improvement in posttreatment $\mathrm{A} 1 \mathrm{C}$ that a patient in the $>75 \%$ adherent group would benefit from, as compared to the reference group (0-25\%) on the $x$-axis. The values on the $x$-axis represent various possible posttreatment $\mathrm{A} 1 \mathrm{C}$ values for the reference group (lowest adherence, $0-25 \%)$. Plotted on the $y$-axis are model estimated differences in post-treatment $\mathrm{A} 1 \mathrm{C}$ values for other adherence groups relative to the reference group. For each of the 3 adherence groups depicted, the $y$-value estimated change in the mean post-A1C compared to the reference group suggests that assuming the same pre- $\mathrm{A} 1 \mathrm{C}$ age group, and $\mathrm{BMI}$, the average for a group with a higher adherence $(>75 \%)$ would have a post-A1C lowered by about 1 . The larger the post-A1C for the low adherence group, the more potential for decreasing by adhering to testosterone therapy. The most significant decrease is in the $>75 \%$ adherence group. The coefficients for the adherence group increase as adherence increases; 0.002 in the $26-50 \%$ group, 0.005 in the $51-75 \%$ group and 0.011 in the $>75 \%$ group. Since the response was transformed to (response=1/ A1C_Post) these increasing coefficients translate to a decrease in post-treatment $\mathrm{A} 1 \mathrm{C}$ as adherence increases, and will also differ depending on the initial $\mathrm{A} 1 \mathrm{C}$ level. The negative values for estimates show that the model predicts that increasing adherence will decrease the estimated post-treatment $\mathrm{A} 1 \mathrm{C}$ values. For the $26-50 \%$ adherence group, the post-treatment $\mathrm{A} 1 \mathrm{C}$ is on average 0.1 to 0.5 lower than those in the $0-25 \%$ group. The post-treatment A1C values are decreased by more in the $51-75 \%$ adherence group $(0.25)$ and even further in the $>75 \%$ adherence group (1.0) compared to the reference group values.

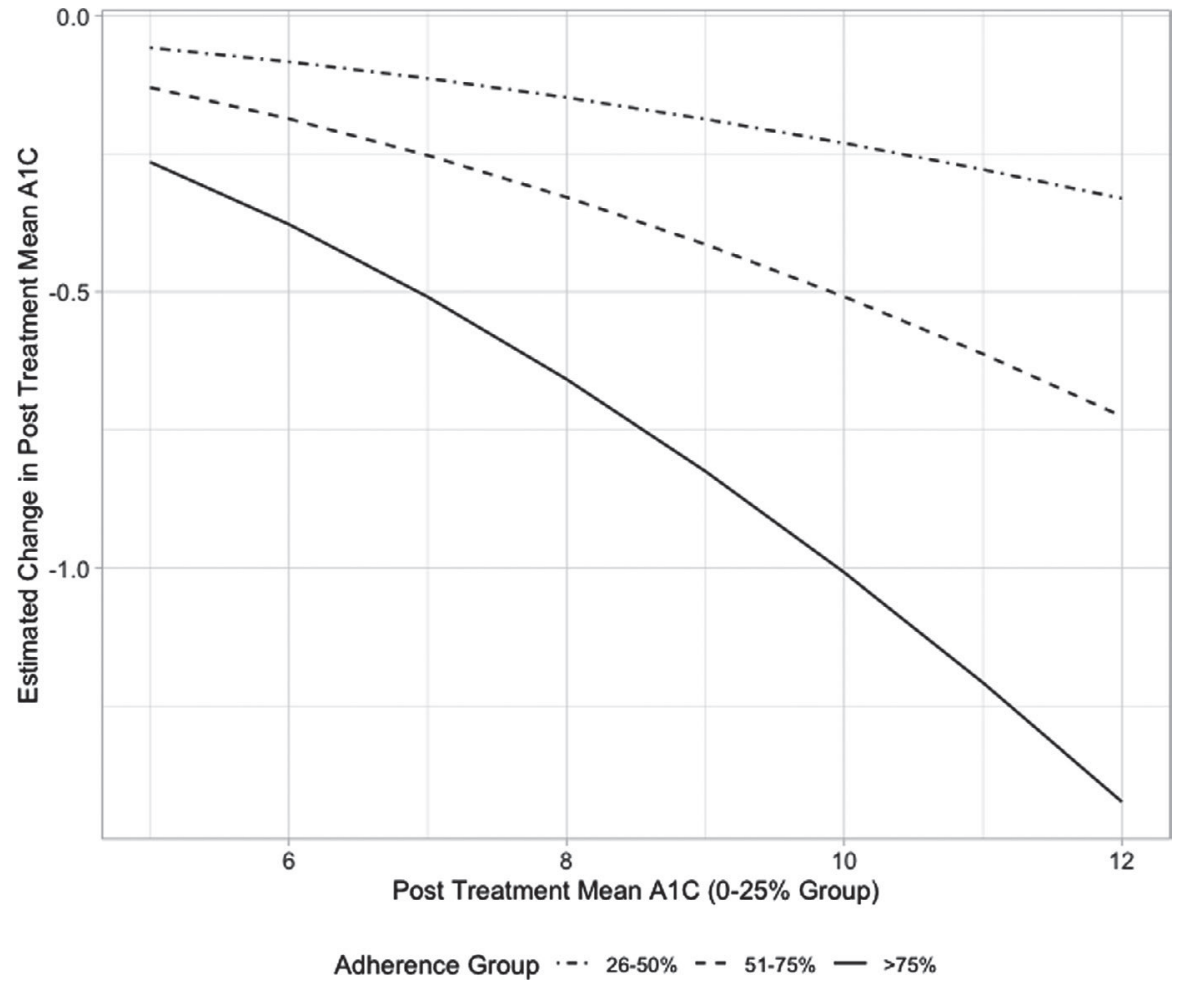

FIGURE 1 Estimated mean change in post-treatment A1C for increased adherence based on post-treatment values of the 0-25\% group, controlling for pre-treatment $\mathrm{A} 1 \mathrm{C}$, age and BMI. Values on the $\mathrm{x}$-axis represent various possible post-treatment $\mathrm{A} 1 \mathrm{C}$ values for group with lowest adherence, $0-25 \%$. Values on the $y$-axis are model estimated differences in post-treatment A1c values for other adherence groups relative to the reference group. All differences control for pre-treatment $\mathrm{A} 1 \mathrm{C}$, age group and $\mathrm{BMI}$ so the comparisons assume populations with the same values for each of these variables. The model predicts that increasing adherence will decrease the estimated post-treatment A1C values. For the $26-50 \%$ adherence group, the post-treatment $\mathrm{A} 1 \mathrm{C}$ is on average 0.1 to 0.5 lower than those in the $0-25 \%$ group. The post-treatment $\mathrm{A} 1 \mathrm{C}$ values decreased more in the $51-75 \%$ adherence group (0.25) and even further in the $>75 \%$ adherence group (2.0) compared to the reference group values 


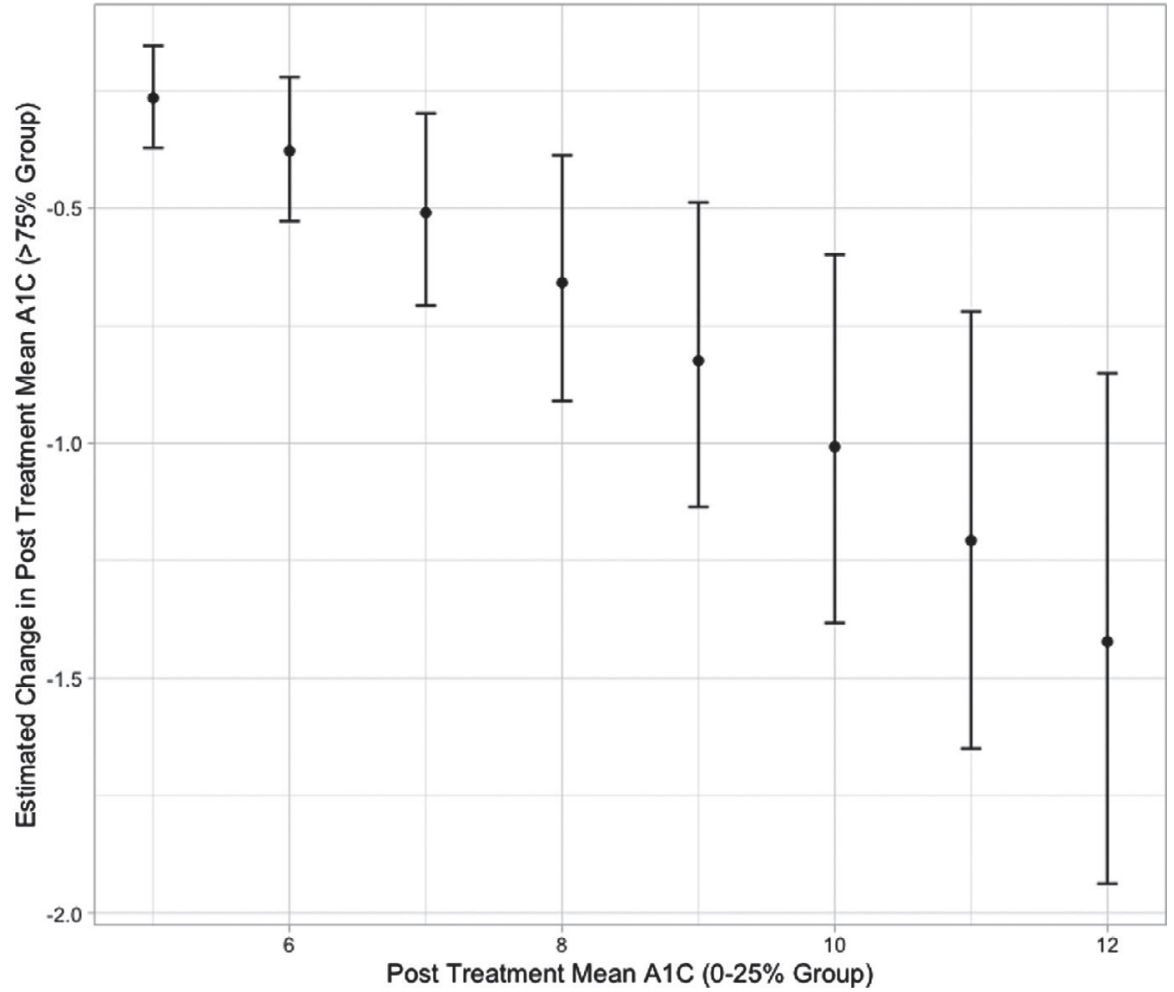

FIGURE 2 Estimated change in post-treatment $\mathrm{A} 1 \mathrm{C}$ for the adherent group (>75\%) compared to $0-25 \%$ posttreatment values, with $95 \%$ confidence bands. These are the estimated decreases due to adherence for various posttreatment $\mathrm{A} 1 \mathrm{C}$ values in the reference groups. The estimates assume the same pre-treatment $\mathrm{A} 1 \mathrm{C}$, age group, and $\mathrm{BMI}$. As an example, consider a population with $0-25 \%$ adherence with a post-treatment A1C of 10 (x-axis). The model estimates the post-treatment would decrease on average by $1 \%$ (to a post-treatment average $\mathrm{A} 1 \mathrm{C}$ of $9 \%, 95 \%$ confidence interval 8.6-9.4\%) if the group improved adherence to $>75 \%$ based on the model
The estimated effect for the adherent group (>75\%) is depicted in more detail in Figure 2. Estimated decreases due to adherence are shown for various post-treatment $\mathrm{A} 1 \mathrm{C}$ values in the reference groups with $95 \%$ confidence bands. Again, the estimates assume the same pre-treatment $\mathrm{A} 1 \mathrm{C}$, age group, and BMI. As an example, consider a population with $0-25 \%$ adherence with a post-treatment A1C of 10 (x-axis). The model estimates the post-treatment would decrease on average by $1 \%$ (to a post-treatment average $A 1 C$ of $9 \%$, 95\% confidence interval 8.6-9.4\%) if the group improved adherence to $>75 \%$ based on the model.

\section{DISCUSSION}

Hypogonadism has been proposed as an independent risk factor for the development of T2DM. ${ }^{4}$ Previous literature has demonstrated controversial evidence regarding whether testosterone therapy should be utilized in mitigating the progression of diabetes, glucose management. and overall $\mathrm{A} 1 \mathrm{C}$ control. In our study of hypogonadal adult male Military Health System beneficiaries with T2DM, we demonstrated interesting findings that suggest that long-term testosterone replacement therapy did, in fact, prove beneficial in a subset of patients with the highest adherence to testosterone replacement therapy. While post-treatment $\mathrm{A} 1 \mathrm{C}$ increased overall for most groups, rising from $6.8 \%$ to $7.1 \%$, the only group to have an $\mathrm{A} 1 \mathrm{C}$ reduction in our study was the group most adherent to long-term testosterone replacement therapy (defined as an adherence of $>75 \%$ with a median $\mathrm{A} 1 \mathrm{C}$ reduction of -0.2 ) compared to the $0-25 \%$ and 26-50\% adherence groups, even when controlling for other factors, including $\mathrm{BMI}$ improvement, age group, and pre-treatment $\mathrm{A} 1 \mathrm{C}$. These changes were statistically significant, suggesting that adherence to testosterone replacement therapy may have independently contributed to lowering $\mathrm{A} 1 \mathrm{C}$ over time.

It has been demonstrated that hypogonadal men with diabetes may benefit from improvements in weight and metabolic index parameters if they are on testosterone therapy. In this 2014 study, investigators found that in the testosterone treatment group, waist circumference, weight, fasting glucose, $\mathrm{A} 1 \mathrm{C}$, blood pressure, lipid profiles, and liver enzymes all improved $(p<0.0001) .{ }^{27}$ However, our statistical model was unique in that it also demonstrated that the higher the initial $\mathrm{A} 1 \mathrm{C}$ at the initiation of testosterone therapy, the higher the potential for the lowering of the patient's $\mathrm{A} 1 \mathrm{C}$ with adherence to testosterone therapy, even when controlling for all other clinical and laboratory variables in our study, including BMI, age, pre-treatment $\mathrm{A} 1 \mathrm{C}$, as shown in Figure 1. When controlling for the same $\mathrm{BMI}$, age, and starting $\mathrm{A} 1 \mathrm{C}$, patients with higher $\mathrm{A} 1 \mathrm{C}$ values when compared to a reference patient on the $\mathrm{x}$-axis have more potential for lowering of their $\mathrm{A} 1 \mathrm{C}$ if they were more compliant with their testosterone therapy based on our model (nearly 2 points for a patient with an $\mathrm{A} 1 \mathrm{C}$ of about $12 \%$ at end of treatment), based on the graph. This may further delineate the effects of testosterone in its potential for mitigating the effects of diabetes in hypogonadal men.

In our study, testosterone replacement therapy also appeared to affect several indices associated with metabolic syndrome. Overall, BMI decreased from $33.9 \pm 0.006$ to $33.5 \pm 0.006$ ( $p=0.052$ ). This $\mathrm{BMI}$ improvement neared but did not reach statistical significance. The change in $\mathrm{A} 1 \mathrm{C}$ was also ultimately associated with a statistically significant decrease in total cholesterol $(p<0.001)$ and an increase in 
$\mathrm{HDL}(p=0.018)$. The change in total and HDL cholesterol levels were statistically significant but not clinically meaningful because they were very small. Although cholesterol measurements were missing for many patients, this was addressed with multiple imputation by chained equations, as addressed in the statistical analysis section, so only complete cases were included to rectify the issue.

Our findings suggesting the benefits of testosterone therapy in treating metabolic indices have been corroborated in prior observational studies. In a 2014 study of hypogonadal obese men treated with testosterone undecanoate (TU), those with T2DM demonstrated a reduction in $\mathrm{BMI}$, waist circumference, and weight, as well as a significant reduction in $\mathrm{A} 1 \mathrm{C} .{ }^{28}$ This impact has also been noted in many articles investigating the role of testosterone in regulating body composition. In a review analyzing data from over 32 retrieved articles, testosterone was found to improve both body composition and metabolic indices. Here, estimated weight loss and waist circumference reduction at 24 months were $-3.50[-5.21 ;-1.80] \mathrm{kg}$ and $-6.23[-7.94 ;-4.76] \mathrm{cm}$, respectively. Testosterone was also associated with a significant reduction in fat, an increase in lean mass, a reduction in fasting glycemia and insulin resistance, and improvements in both systolic and diastolic blood pressure. Further, when only considering hypogonadal men (total $\mathrm{T}<12 \mathrm{~mol} / \mathrm{l}$ ) in the review, a reduction of total cholesterol was noted, which is consistent with our findings. ${ }^{29}$

Similar findings on the impact of testosterone on metabolic syndrome indices have also found in other studies that focused solely on hypogonadal men. ${ }^{30}$ In this observational study, 5 years of testosterone treatment led to marked and significant decrease in body weight, waist circumference and body mass index. ${ }^{31}$ Even when focusing solely on elderly men with hypogonadism and erectile dysfunction, testosterone undecanoate similarly led to significant improvements in the same obesity parameters. ${ }^{32}$

The reduction in metabolic risk is likely multifactorial. Obesity leads to low total testosterone levels by changing sex hormone binding globulin (SHBG) levels, decreasing luteinizing hormone levels, and causing adipocyte dysfunction, androgen resistance, and insulin resistance. Testosterone therapy in turn reduces lipoprotein lipase and triglyceride activity, which results in increased lean body mass and decreased fat mass. ${ }^{33}$ These reductions in body weight and waist circumference in men with obesity and hypogonadism who are treated with testosterone have been established. ${ }^{34}$ And yet, while these benefits for the parameters associated with glycemia and metabolic syndrome are well-established, testosterone supplementation in hypogonadal men appears to improve additional health benefits, including improvements in renal function and other lifestyle markers including fatigue, erectile dysfunction, libido, and motivation with testosterone therapy. ${ }^{35-37}$

However, there are concerns among experts regarding testosterone replacement therapy which include ongoing, extensive debates about its benefit-risk ratio and potential CVD risks, especially in aging men. Due to two studies which associated testosterone therapy with increased risk of myocardial infarction and stroke, the U.S. Food and Drug Administration issued a warning regarding its use in
$2014 .^{38,39}$ This warning led to a decrease in the dispensing of testosterone prescriptions. ${ }^{40}$ One study showed that non-calcified coronary artery plaque volume was significantly increased among elderly hypogonadal men after one year of testosterone therapy, though correlation with increased CVD risk is still unknown. ${ }^{41}$ However, another study that focused on long-term testosterone therapy and cardiometabolic function demonstrated that long-term TU was associated with reduced mortality related to $\mathrm{CV}$ disease. ${ }^{42}$ Again, while another study that focused on elderly men with hypogonadism on testosterone therapy for 5 years found no evidence that long-term TU increases the risk of prostate carcinoma, ${ }^{32}$ other cardiac specific findings found in some prior studies warrant further well-powered studies to better determine the long-term CV risks.

Due to the benefits of TU on metabolic indices in men with obesity and hypogonadism, clinical practice guidelines do support TU as an adjunct to lifestyle intervention. ${ }^{43}$ However, the widespread utilization of testosterone for weight reduction and metabolic improvement in men with obesity and hypogonadism remains controversial. ${ }^{37}$ This discrepancy may be due to relatively lower efficacy and the transient nature of metabolic and gonadal improvement when comparing TU with interventions such as bariatric surgery, as some improvements have been noted to revert to baseline after cessation of testosterone therapy. ${ }^{44}$

Nevertheless, testosterone administration with high therapy adherence may be a useful tool to improve $\mathrm{HbA} 1 \mathrm{C}$, lipid profile, and $\mathrm{BMI}$ among patients with hypogonadism and T2DM. While previous literature has demonstrated evidence regarding testosterone therapy's ability to mitigate the progression of diabetes, overall $\mathrm{A} 1 \mathrm{C}$ control, and glucose management in men with hypogonadism and type 2 diabetes, it has been unclear as to whether the degree of improvements in glycemic control can vary depending on the extent to which a patient is compliant with their testosterone therapy, or if adherence to one therapy is a predictor for adherence to other behaviors that improve both diabetes and hypogonadism - particularly diet, exercise, sleep, and consistency with other medications. There is limited literature evaluating the effect of adherence to testosterone replacement therapy on both diabetes and hypogonadism-related outcomes. One study evaluating men on topical testosterone found that fatigue, depression, erectile dysfunction, insomnia, and osteoarthritis improved in the group adherent to topical testosterone, but did not reach statistical significance. ${ }^{45}$ To our knowledge, then, at the time of this publication, there are no other studies specifically evaluating adherence to topical and intramuscular testosterone preparations and its direct effects on glycemic control in men with hypogonadism and diabetes.

Our study is also unique in its contribution to the current scientific literature in three ways. Firstly, our findings showed that while improvement in BMI did trend toward statistical significance, it did not actually reach significance, and yet, adherence to testosterone was nevertheless found to be associated with marked and statistically significant improvement in $\mathrm{A} 1 \mathrm{C}$. Secondly, even when adjusting and controlling for $\mathrm{BMI}$, pre-treatment $\mathrm{A} 1 \mathrm{C}$, and age, our findings showed that the patients with the highest adherence to testosterone 
were the most likely to benefit from testosterone therapy. Lastly, the patients with the highest $\mathrm{A} 1 \mathrm{C}$ at initial treatment were most likely to benefit from testosterone if they were the most adherent to testosterone. When considering that patients with the highest $\mathrm{A} 1 \mathrm{C}$ values would not be expected to practice behaviors that improve diabetes in general (ie, a healthy diet, exercise, healthy sleep habits), and still noting the preferential improvement in $\mathrm{HbA1C}$ with testosterone adherence in this group, it follows that testosterone may warrant more clinical attention in its ability to improve diabetes management in hypogonadal males.

\section{1 | Study Limitations}

There are several study limitations which should be addressed. One limitation was determining if these patients were truly hypogonadal. While testosterone replacement therapy can benefit men with true hypogonadism, the diagnosis of hypogonadism is challenging. There are debates about which laboratory assays are most appropriate for it and what constitutes their normal ranges. ${ }^{46}$ There is also a level of subjectivity when accounting for patient symptoms in the diagnostic determination, and research studies have included participants with wide ranges of serum testosterone. ${ }^{47}$ There is more than one clinical guideline in place regarding the diagnosis and initiation of treatment in hypogonadal patients, which has led to wide clinician variability in the diagnosis and management of these patients. ${ }^{48}$ This challenge is also reflected in the clinical practice variations across different guidelines and medical societies regarding the management of hypogonadism. In a recent literature review compiling data from 9 major medical societies, it was reported that discrepancies were noted both for the diagnostic workup measures of total and free testosterone, and follow-up or monitoring guidelines for total testosterone levels in men with hypogonadism or late-onset hypogonadism. ${ }^{49}$

As a result, one main limitation of our study involved the lack of diagnostic uniformity and laboratory information regarding the diagnosis and management of hypogonadism. It is not completely clear what cutoff levels of testosterone levels were used, and which clinical characteristics and symptoms clinicians used to diagnose overt hypogonadism in our diabetic men. Thus, there was likely some clinical variability in the diagnosis of hypogonadism in our study, based on the clinical discretion of the provider, the symptomatology of the patients, and lab cutoffs. Being a retrospective study in nature, patients were included for analysis if they met the inclusion criteria based on ICD codes for hypogonadism and type 2 diabetes, and our study data was collected from there. Due to the multiple lab assays seen throughout the study period, the ambiguous cutoff values to determine hypogonadism diagnosis, and the nature of the inclusion criteria based on ICD codes and clinician discretion, no testosterone lab values were used in the analysis of our data. While this is a challenge for any study involving hypogonadal men, it nevertheless references an intrinsic limitation of our study given the targeted patient population, which may cloud the results, skewing the apparent risk/ benefit ratio of testosterone replacement therapy.
Regarding the role that labs play in the diagnosis of hypogonadism, it is important to note that an accurate measurement of total testosterone is one of the most difficult hurdles to overcome in the diagnosis of hypogonadism, with variables related to both the different measuring assays and the different reference ranges used. ${ }^{50}$ Many studies have attempted to more accurately quantify total serum testosterone levels, but variability related to weight, age, lifestyle differences, diet, and overall lack of consensus on normal lab values for total testosterone continues to make the utility of testosterone in the setting of the diagnosis of hypogonadism difficult. ${ }^{48}$ Ideally, the use of free testosterone would have been included in our study. However, given the lack of free testosterone levels collected during our study period, we found that this was not often used in the diagnosis or management of hypogonadism during the follow up period by many practitioners. ${ }^{51}$ For this reason, free testosterone evaluations may not have been taken into account in certain patient populations in which standard of care would now necessitate the utility of this lab. An example of this would be in men with any alterations in SHBG. ${ }^{52}$

Another limitation is that we did not delineate which testosterone formulations were given to the patients. We also do not know if prescriptions for testosterone were given inappropriately, for example in a setting in which a patient received a prescription after only 1 or no testosterone laboratory tests were drawn. Patients may have had ill-timed testosterone laboratory evaluations, meaning that the lab draw may not have been taken in the early morning or close to the time the patients typically awoke, which would be optimal for testosterone testing. We made the assumption that patients who filled their prescriptions were using them because of their long follow-up duration, but it is possible this assumption was not always correct. We did not collect cardiovascular safety data on patients on long-term testosterone therapy, and further studies are needed to better elucidate these risks. An inherent limitation with $\mathrm{BMI}$ is that it does not take into account muscle vs fat weight and increased lean muscle mass is associated with higher insulin sensitivity. ${ }^{53}$

Lastly, in order to establish objective, concrete lab and metabolic parameter cutoffs for the study, and to more easily identify potential lab errors warranting exclusion, established thresholds for $\mathrm{HbA} 1 \mathrm{C}$ lab values ranging from $4 \%-12 \%$ were selected as within threshold for our study. While this could help identify potential lab errors outlying this range, this also could have led to a selection bias in our participants, if, for example, a patient had a legitimate $\mathrm{HbA} 1 \mathrm{C}$ value $>12 \%$. This could have been avoided had the inter and intra-assay coefficients of variation (CV\%) of each method employed been reported, but this data was not collected for review.

\section{2 | Conclusion}

Our study contributes to the current body of evidence in the field, because our data demonstrate that even when controlling for other 
clinical and laboratory data including BMI, pre-treatment $\mathrm{A} 1 \mathrm{C}$, and age, testosterone therapy positively impacted glycemic control in those diabetes patients who had the highest compliance with therapy as evidenced by most adherence. Further, our model was unique in that it demonstrated that the higher the initial $\mathrm{A} 1 \mathrm{C}$ at the initiation of testosterone therapy, the higher the potential for the lowering of the patient's $\mathrm{A} 1 \mathrm{C}$ with $>75 \%$ adherence, even when controlling for $\mathrm{BMI}$, age, and pre-treatment $\mathrm{A} 1 \mathrm{C}$. There was a statistically significant reduction in $\mathrm{A} 1 \mathrm{C}$ for patients with higher adherence to testosterone replacement therapy versus an increase in $\mathrm{A} 1 \mathrm{C}$ for those with less adherence. This supports its merit as a tool in improving $\mathrm{HbA1C}$ among these patients with hypogonadism and DM. All groups showed some reduction in BMI, however, which may further indicate that testosterone therapy may affect $\mathrm{A} 1 \mathrm{C}$ independent of weight loss.

\section{CONFLICT OF INTEREST}

The authors have nothing to disclose.

\section{AUTHOR CONTRIBUTIONS}

CRJ, JW, PGC, EM, LMJ, JLW and SDG, analyzed the data and wrote the paper. AR and RXS performed the research acquired the data, analyzed the data and wrote the paper.

\section{DATA AVAILABILITY STATEMENT}

The data that support the findings of this study are available from the Defense Health Agency but restrictions may apply to the availability of these data, which were used under a data sharing agreement for the current study, and so are not publicly available. Data are however available from the authors upon reasonable request and with permission of the Defense Health Agency.

\section{ORCID}

Craig R. Jenkins (D) https://orcid.org/0000-0002-7604-4873

Sky D. Graybill (D) https://orcid.org/0000-0002-0556-6754

\section{REFERENCES}

1. Cowie CC, Rust KF, Ford ES, et al. Full accounting of diabetes and pre-diabetes in the U.S. population. Diabetes Care. 2009;32:287294.in 1988-1994 and 2005-2006.

2. Ligthart S, van Herpt TT, Leening MJ, et al. Lifetime risk of developing impaired glucose metabolism and eventual progression from prediabetes to type 2 diabetes: a prospective cohort study. Lancet Diabetes Endocrinol. 2016;4:44-51.

3. Dhindsa S, Prabhakar S, Sethi M, Bandyopadhyay A, Chaudhuri A, Dandona P. Frequent occurrence of hypogonadotropic hypogonadism in type 2 diabetes. J Clin Endocrinol Metab. 2004;89: 5462-5468.

4. Stellato RK, Feldman HA, Hamdy O, Horton ES, McKinlay JB. Testosterone, sex hormone-binding globulin, and the development of type 2 diabetes in middle-aged men: prospective results from the Massachusetts male aging study. Diabetes Care. 2000;23:490-494.

5. Kapoor D, Goodwin E, Channer KS, Jones TH. Testosterone replacement therapy improves insulin resistance, glycaemic control, visceral adiposity and hypercholesterolaemia in hypogonadal men with type 2 diabetes. Eur J Endocrinol. 2006;154:899-906. o. Jones I $\mathrm{H}$, Arver $د$, Benre rivı, et al. Iestosterone repiacement in hypogonadal men with type 2 diabetes and/or metabolic syndrome (the TIMES2 study). Diabetes Care. 2011;34:828-837.

7. Dhindsa S, Ghanim H, Batra M, et al. Insulin resistance and inflammation in hypogonadotropic hypogonadism and their reduction after testosterone replacement in men with type 2 diabetes. Diabetes Care. 2016;39:82-91.

8. Gianatti EJ, Dupuis P, Hoermann R, et al. Effect of testosterone treatment on glucose metabolism in men with type 2 diabetes: a randomized controlled trial. Diabetes Care. 2014;37:2098-2107.

9. Grossmann M, Hoermann R, Wittert G, Yeap BB. Effects of testosterone treatment on glucose metabolism and symptoms in men with type 2 diabetes and the metabolic syndrome: a systematic review and meta-analysis of randomized controlled clinical trials. Clin Endocrinol (Oxf). 2015;83:344-351.

10. Corona G, Monami M, Rastrelli G, et al. Type 2 diabetes mellitus and testosterone: a meta-analysis study. Int J Androl. 2011;34:528-540.

11. Haider KS, Haider A, Saad A, et al. Remission of type 2 diabetes following long-term treatment with injectable testosterone undecanoate in patients with hypogonadism and type 2 diabetes: 11year data from a real-world registry study. Diabetes Obes Metab. 2020;22:2055-2068.

12. Yassin A, Haider A, Haider KS, et al. Testosterone therapy in men with hypogonadism prevents progression from prediabetes to Type 2 diabetes: Eight-year data from a registry study. Diabetes Care. 2019;42:1104-1111.

13. Giagulli VA, Castellana M, Carbone MD, et al. Weight loss more than glycemic control may improve testosterone in obese type 2 diabetes mellitus men with hypogonadism. Andrology. 2020;8:654-662.

14. Grams J, Garvey WT. Weight loss and the prevention and treatment of type 2 diabetes using lifestyle therapy, pharmacotherapy, and bariatric surgery: mechanisms of action. Curr Obes Rep. 2015;4:287-302.

15. American Diabetes Association. Comprehensive medical evaluation and assessment of comorbidities: standards of medical care in diabetes-2018. Diabetes Care. 2018;41:S28-S37.

16. Paris RM, Bedno SA, Krauss MR, Keep LW, Rubertone MV. Weighing in on type 2 diabetes in the military: characteristics of U.S. military personnel at entry who develop type 2 diabetes. Diabetes Care. 2001;24:1894-1898.

17. Araujo AB, Dixon JM, Suarez EA, Murad MH, Guey LT, Wittert GA. Clinical review: endogenous testosterone and mortality in men: a systematic review and meta-analysis. J Clin Endocrinol Metab. 2011;96:3007-3019.

18. Janghorbani M, Van Dam RM, Willett WC, Hu FB. Systematic review of type 1 and type 2 diabetes mellitus and risk of fracture. Am J Epidemiol. 2007;166:495-505.

19. Tunceli K, Bradley CJ, Nerenz D, Williams LK, Pladevall M, Elston LJ. The impact of diabetes on employment and work productivity. Diabetes Care. 2005;28:2662-2667.

20. Knowler WC, Fowler SE, Hamman RF, et al. 10-year follow-up of diabetes incidence and weight loss in the Diabetes Prevention Program Outcomes Study. Lancet. 2009;374:1677-1686.

21. Diabetes Prevention Program Research Group. Long-term effects of lifestyle intervention or metformin on diabetes development and microvascular complications over 15-year follow-up: the Diabetes Prevention Program Outcomes Study. Lancet Diabetes Endocrinol. 2015;3:866-875.

22. Hosmer DW, Lemeshow S, Sturdivant RX. Applied Logistic Regression. New York, NY: John Wiley \& Sons; 2013.

23. O'Connell NS, Dai L, Jiang Y, et al. Methods for analysis of pre-post data in clinical research: a comparison of five common methods. $J$ Biom Biostat. 2017;8:1-8.

24. Box GEP, Cox DR. An analysis of transformations. J R Stat Soc Ser B Methodol. 1964;26:211-243. 
25. van Buuren S, Groothuis-Oudshoorn K. MICE: multivariate imputation by chained equations in R. J Stat Softw. 2010;45:1-67.

26. Rubin D. Multiple Imputation for Nonresponse in Surveys. New York, NY: John Wiley \& Sons; 1987.

27. Haider A, Yassin A, Doros G, Saad F. Effects of long-term testosterone therapy on patients with "diabesity": Results of observational studies of pooled analyses in obese hypogonadal men with type 2 diabetes. Int J Endocrinol. 2014;2014:682515.

28. Haider A, Saad F, Doros G, Gooren L. Hypogonadal obese men with and without diabetes mellitus type 2 lose weight and show improvement in cardiovascular risk factors when treated with testosterone: an observational study. Obes Res Clin Pract. 2014;8:e339-e349.

29. Corona G, Giagulli VA, Maseroli E, et al. Testosterone supplementation and body composition: results from a meta-analysis study. Eur J Endocrinol. 2016;174:R99-R116.

30. Zitzmann M, Saad F. Differential effects of intramuscular testosterone undecanoate in hypogonadal men on body weight and waist circumference during 16 years of treatment. In: The Endocrine Society's 95th Annual Meeting and Expo. June 15-18.2013; San Francisco, CA. Abstract.

31. Yassin A, Doros G. Testosterone therapy in hypogonadal men results in sustained and clinically meaningful weight loss. Clin Obes. 2013;3:73-83.

32. Yassin DJ, Doros G, Hammerer PG, Yassin A. Long-term testosterone undecanoate treatment in elderly men with late-onset hypogonadism and erectile dysfunction reduces obesity parameters and improves metabolic syndrome and health-related quality of life. $J$ Sex Med. 2014;11:1567-1576.

33. Traish AM. Testosterone and weight loss: the evidence. Curr Opin Endocrinol Diabetes Obes. 2014;21:313-322.

34. Hackett G, Cole N, Bhartia M, Kennedy D, Raju J, Wilkinson P. BLAST Study Group. Testosterone replacement therapy improves metabolic parameters in hypogonadal men with type 2 diabetes but ot in men with coexisting depression: the BLAST study. J Sex Med. 2014;11(3):840-856.

35. Yassin A, Almehmadi $\mathrm{Y}$, Alwani M, et al. Long-term Testosterone Therapy Improves Renal Function in Men with Hypogonadism: A Real-life Prospective Controlled Registr. J Clin Nephrol Res. 2020;7(1):1095.

36. Pexman-Fieth C, Behre HM, Morales A, Kan-Dobrosky N, Miller MG. A 6-month observational study of energy, sexual desire, and body proportions in hypogonadal men treated with a testosterone 1\% gel. Aging Male. 2014;17:1-11.

37. Corona G, Torres LO, Maggi M. Testosterone therapy: What we have learned from trials. J Sex Med. 2020;17:447-460.

38. Vigen R, O'Donnell $\mathrm{Cl}$, Barón $\mathrm{AE}$, et al. Association of testosterone therapy with mortality, myocardial infarction, and stroke in men with low testosterone levels. JAMA. 2013;310:1829-1836.

39. Finkle WD, Greenland S, Ridgeway GK, et al. Increased risk of nonfatal myocardial infarction following testosterone therapy prescription in men. PLoS One. 2014;9:e85805.

40. Baillargeon J, Kuo YF, Westra JR, Urban RJ, Goodwin JS. Testosterone prescribing in the United States, 2002-2016. JAMA. 2018;320:200-202
41. Budoff MJ, Ellenberg SS, Lewis CE, et al. Testosterone treatment and coronary artery plaque volume in older men with low testosterone. JAMA. 2017;317:708-716.

42. Traish AM, Haider A, Haider KS, Doros G, Saad F. Long-term testosterone therapy improves cardiometabolic function and reduces risk of cardiovascular disease in men with hypogonadism: a real-life observational registry study setting comparing treated and untreated (control) groups. J Cardiovasc Pharmacol Ther. 2017;22:414-433.

43. Garvey WT, Mechanick JI, Brett EM, et al. American Association Of Clinical Endocrinologists And American College Of Endorcrinology Comprehensive Clinical Practice Guidelines For Medical Care Of Patients With Obesity. Endocr Pract. 2016;22(Suppl 3):1-203.

44. Seftel A. Re: effects of testosterone undecanoate on cardiovascular risk factors and atherosclerosis in middle-aged men with late-onset hypogonadism and metabolic syndrome; results from a 24-month, randomized, double-blind, placebo-controlled study. J Urol. 2011;185(2):634.

45. Grabner M, et al. Topical Testosterone Therapy Adherence and Outcomes Among Men with Primary or Secondary Hypogonadism. J Sex Med. 2018;15(2):148-158.

46. Bhasin S, Ellenberg SS, Storer TW, et al. Effect of testosterone replacement on measures of mobility in older men with mobility limitation and low testosterone concentrations: secondary analyses of the Testosterone Trials. Lancet Diabetes Endocrinol. 2018;6:879-890.

47. Wu FC, Tajar A, Beynon JM, et al. Identification of late-onset hypogonadism in middle-aged and elderly men. $N$ Engl J Med. 2010;363:123-135.

48. Defeudis $G$, Mazzilli R, Gianfrilli D, Lenzi A, Isidori AM. The CATCH checklist to investigate adult-onset hypogonadism. Andrology. 2018;6:665-679.

49. Giagulli VA, Castellana M, Lisco G, Triggiani V. Critical evaluation of different available guidelines for late-onset hypogonadism. Andrology. 2020;8(6):1628-1641.

50. Trost LW, Mulhall JP. Challenges in testosterone measurement, data interpretation, and methodological appraisal of interventional trials. J Sex Med. 2016;13:1029-1046.

51. Khera M, Broderick GA, Carson CC 3rd, et al. Adult-onset hypogonadism. Mayo Clin Proc. 2016;91:908-926.

52. Bhasin S, Brito JP, Cunningham GR, et al. Testosterone therapy in men with hypogonadism: an Endocrine Society clinical practice guideline. J Clin Endocrinol Metab. 2018;103:1715-1744.

53. Srikanthan P, Karlamangla AS. Relative muscle mass is inversely associated with insulin resistance and prediabetes. Findings from the third National Health and Nutrition Examination Survey. J Clin Endocrinol Metab. 2011;96:2898-2903.

How to cite this article: Jenkins CR, Rittel A, Sturdivant RX, et al. Glycemic benefits with adherence to testosterone therapy in men with hypogonadism and type 2 diabetes mellitus. Andrology. 2021;9:1076-1085. https://doi.org/10.1111/ andr.12990 